\title{
Foville Syndrome Due to Pontine Hemorrhage and Pontine Infarct: Case Series
}

\author{
$\underline{\text { Imran } I^{1}, \text { Syahrul S}^{2} \text {, Nurul Fajri }}{ }^{3}$ \\ 1,2,3 Neurology Department, Faculty of Medicine, Universitas Syiah Kuala, Dr. Zainoel Abidin General \\ Hospital, Banda Aceh, Indonesia
}

\begin{abstract}
:
Foville Syndrome is a rare clinical feature of stroke or brain hemorrhage. Few cases have been reported worldwide particularly in Asian, Indonesia, Aceh. Patient 1: We report the case of a female 64 years old patient, was admitted in the Neurology Department of Dr. Zainoel Abidin General Hospital, Banda Aceh, Indonesia, for an abrupt onset of right body side weakness two day before admission. That weakness was preceded by acute headaches and rotatory vertigo with vomiting. with hypertension history since 5 years. Physical examination found paralysis of right oculomotor movements with intact response of pupils to light, right trochlear nerve palsy, left ipsilateral facial nerve palsy, and right hemiparesis which constitute of Foville syndrome. The brain CT scan shown in the right pontine hypodensity area is pontine infarct. Etiological investigations done to chronic hypertension. Patient received antiplatelet therapy and control of vascular risk factors for secondary prevention (hypertension theraphy), and physiotherapy as treatment with clinical improvement. And she has been discharge 8 days later. Patient 2: 45year-old right-handed female, was admitted in the Neurology Department of Dr. Zainoel Abidin General Hospital, Banda Aceh, Indonesia, for an abrupt onset of right body side weakness two day before admission. That weakness was preceded by acute headaches and rotatory vertigo with vomiting. with hypertension history since 5 years. Physical examination found paralysis of right oculomotor movements with intact response of pupils to light, right trochlear nerve palsy, left ipsilateral facial nerve palsy, and right hemiparesis which constitute of Foville syndrome. The brain CT scan shown spontaneous hemorrhage in the left pontine's. Etiological investigations done to chronic hypertension. Patient received therapy control of vascular risk factors for secondary prevention (hypertension theraphy), and physiotherapy as treatment with clinical improvement. And she has been discharge 10 days later. The diagnosis and management of brainstem stroke bring a considerable burden to the healthcare system, the patient, the family members, and the society at large. The slow increase in the global burden of stroke has been steadily increasing.
\end{abstract}

Keywords:

foville syndrome; pontine hemorrhage; pontine infarct

\section{Introduction}

Stroke is one of the leading causes of mortality and morbidity in the world. Pontine strokes, in particular, are catastrophic. Stroke disease is the most dominant health complication resulting in death and disability (Sari, 2020). Stroke is a global epidemic, and hence, it is not limiting to developed (Ekerikevwe, 2020). Clinical presentation of a pontine infarction can vary, ranging from the classical crossed syndrome (ipsilateral cranial nerve palsy and contralateral motor and/or sensory impairment) to the less common pure motor hemiparesis or hemiplegia or pure sensory stroke. A majority of stroke events and their complications can be prevented with proper care. This activity outlines the presentation, evaluation, and management of infarction in the pons and highlights the role of the healthcare team in evaluating and treating patients with this condition (Jillella, 2021). 
Foville syndrome is a rare inferior medial pontine syndrome first characterized in 1858 by anatomist and psychiatrist Achille Louis Francois Foville. In his paper, "Notes on a Littleknown Paralysis of Eye Muscles and Its Relation to the Anatomy and Physiology of the Pons". (Naqvi, 2021).

\section{Patient and Observation}

Patient 1: We report the case of a female 64 years old patient, was admitted in the Neurology Department of Dr. Zainoel Abidin General Hospital, Banda Aceh, Indonesia, for an abrupt onset of right body side weakness two day before admission. That weakness was preceded by acute headaches and rotatory vertigo with vomiting with hypertension history since 5 years. Physical examination found paralysis of right oculomotor movements with intact response of pupils to light, right trochlear nerve palsy, left ipsilateral facial nerve palsy, and right hemiparesis which constitute of Foville syndrome. The brain CT scan shown in the right pontine bypodensity area is pontine infarct. Etiological investigations done to chronic hypertension. Patient received antiplatelet therapy and control of vascular risk factors for secondary prevention (hypertension theraphy), and physiotherapy as treatment with clinical improvement. And she has been discharge 8 days later.
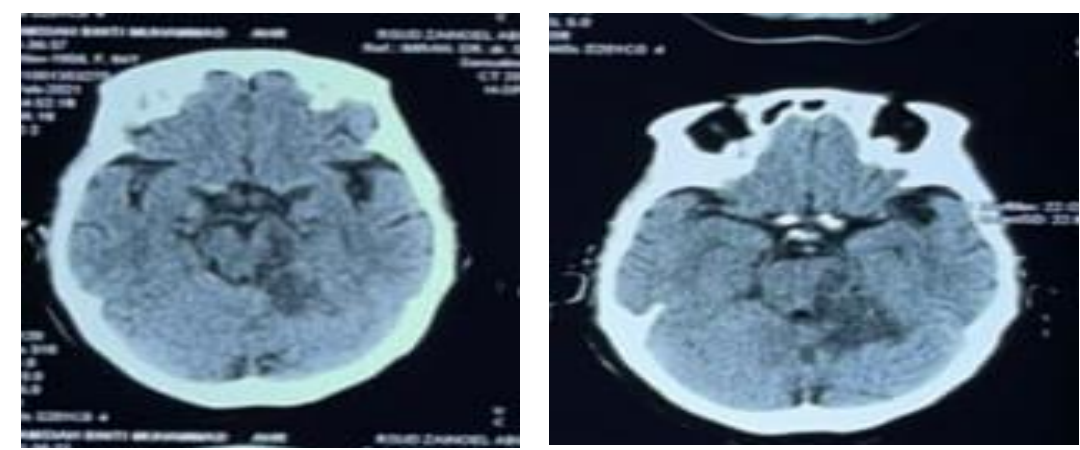

Figure 1. Axial Brain CT Scan Showing the Right Pontine Hypodensity Area is Pontine Infarct

Patient 2: 45-year-old right-handed female, was admitted in the Neurology Department of Dr. Zainoel Abidin General Hospital, Banda Aceh, Indonesia, for an abrupt onset of right body side weakness two day before admission. That weakness was preceded by acute headaches and rotatory vertigo with vomiting with hypertension history since 5 years. Physical examination found paralysis of right oculomotor movements with intact response of pupils to light, right trochlear nerve palsy, left ipsilateral facial nerve palsy, and right hemiparesis which constitute of Foville syndrome. The brain CT scan shown spontaneous hemorrhage in the left pontine's. Etiological investigations done to chronic hypertension. Patient received therapy control of vascular risk factors for secondary prevention (hypertension theraphy), and physiotherapy as treatment with clinical improvement. And she has been discharge 10 days later.
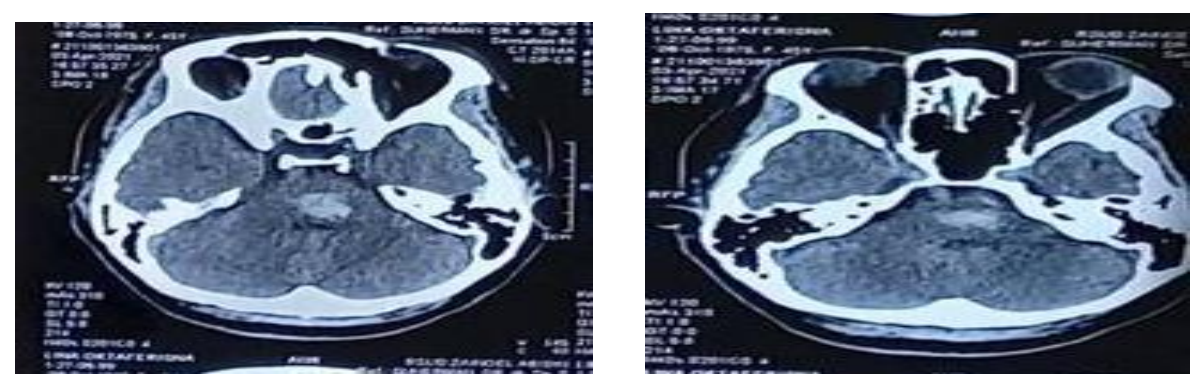

Figure 2. Axial Brain CT Scan Showing Spontaneous Hemorrhage in the Left Pontine's 


\section{Discussion}

The brainstem is composed of the midbrain, the pons, and the medulla oblongata, situated in the posterior part of the brain. It is a connection between the cerebrum, the cerebellum, and the spinal cord. Embryologically, it develops from the mesencephalon and part of the rhombencephalon, all of which originate from the neural ectoderm. The brainstem is organized internally in three laminae: tectum, tegmentum, and basis. Gray matter in the brainstem is found in clusters all along the brainstem to forming mostly the cranial nerve nuclei, the pontine nuclei, and the reticular formation. White matter in the form of various ascending and descending tracts can be found mainly in the basis lamina, which is the most anterior part. The brainstem is responsible for multiple critical functions, including respiration, cardiac rhythm, blood pressure control, consciousness, and sleep-wake cycle. The cranial nerve nuclei that are present in the brainstem have a crucial role in vision, balance, hearing, swallowing, taste, speech, motor, and sensory supply to the face. The white matter of the brainstem carries most of the signals between the brain and the spinal cord and helps with its relay and processing (Jesus, 2021).

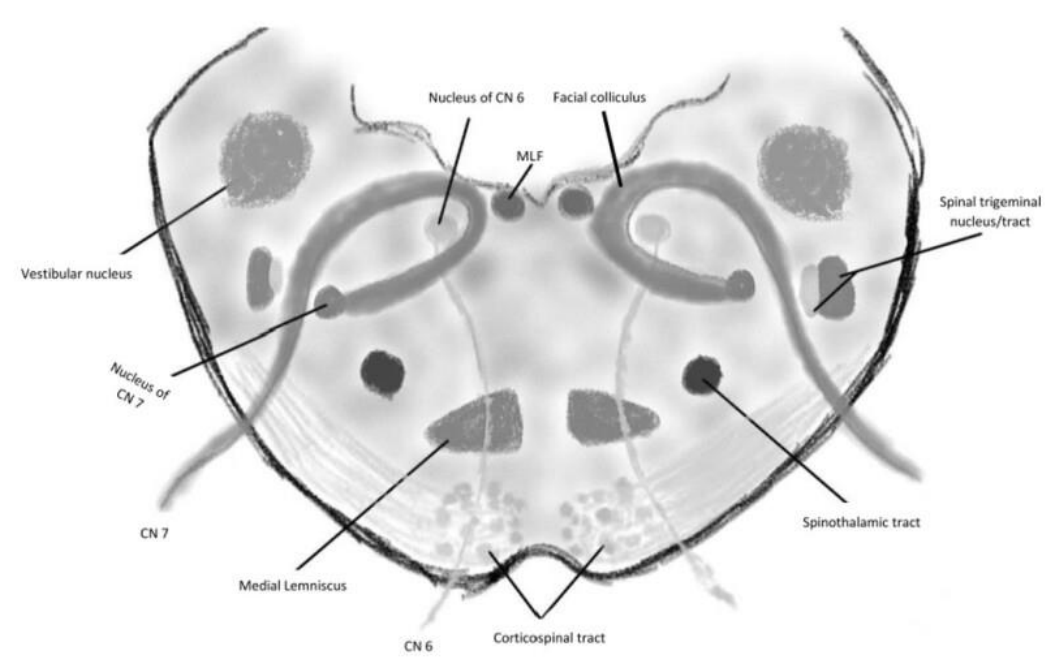

Figure 3: Anatomy of Foville Syndrome at the Level of the inferomedial pons ${ }^{2}$

Ischemic stroke risk increases with age, and hence a typical presentation of a pontine infarction would be an elderly individual with a history of chronic conditions like hypertension, diabetes, dyslipidemia, or history of heart disease. However, pontine infarction can also occur in younger individuals with vascular disorders like CADASIL (cerebral autosomal dominant arteriopathy with subcortical infarcts and leukoencephalopathy), hypercoagulable states, vertebral artery dissections et cetera. Establishing the time of onset of stroke symptoms heavily determines the treatment strategy (thrombolytics vs. thrombectomy). The clinical presentation of a pontine infarct depends on the anatomical/arterial territories involved and are clinically classified as below (Wei, Y. 2020).

Ventro-caudal pontine infarction is caused due to decreased blood flow in the paramedian perforating arteries arising from the basilar artery. Affected individuals have contralateral motor hemiparesis or hemiplegia due to the large infarcts of the unilateral corticospinal tract. Ipsilateral abducens and/or facial nerves palsy can occur secondary to the involvement of the nerve fibers and nucleus, respectively. Lateral spinothalamic tract infarction leads to decreased pain and temperature sensation on the opposite side of the body. Clinically, the presenting symptoms can be grouped into Millard-Gubler syndrome 
(contralateral hemiparesis, ipsilateral abducens, and facial nerve palsy), Foville syndrome (conjugate gaze palsy in addition to the features of Millard-Gubler syndrome) and Raymond syndrome (contralateral body hemiparesis, ipsilateral abducens palsy without facial nerve palsy) (Saposnik, G. 2008; Elyas, AE. 2011).

Mid-pontine base infarction is also due to decreased blood flow in the paramedian arteries or the short circumferential arteries arising from the basilar artery. Presenting symptoms depend on the extent of involvement of various structure within the pons. For instance, ipsilateral ataxia is due to the infarction of pontine nuclei; sensorimotor weakness of the ipsilateral face is due to the trigeminal nerve fibers' involvement and contralateral hemiparesis due to the corticospinal tract. Various combination of these symptoms result in distinct syndromes like pure motor hemiparesis (lacunar infarcts of the corticospinal tract), ataxic hemiparesis (lacunar infarcts of the pontine nuclei), dysarthria-clumsy hand syndrome (dysarthria, dysphagia, impaired dexterity and weakness of hand) and rare presentations like dysarthria-dysmetria and dysarthria-facial paresis (Saposnik, G. 2008; Elyas, AE. 2011).

Tegmental pontine syndrome can affect various structures, including cranial nerve (trigeminal, abducens, facial, and vestibulocochlear) nuclei, medial lemniscus, medial longitudinal fasciculus, respiratory centers, and the pontine reticular formation. Obstruction of blood flow in the anterior inferior or superior cerebellar arteries causes the rostral pontine syndrome. Symptoms of this syndrome include-ipsilateral facial sensory disturbance and masticator paralysis (trigeminal nuclei), impaired blinking (tectospinal tract); contralateral hemisensory loss (lateral spinothalamic tract and medial lemniscus); and ipsilateral hemiataxia (superior cerebellar peduncle). The caudal pontine syndrome is caused due to decreased blood flow in the short circumferential or the anterior inferior cerebellar artery. Clinically, they can present with- ipsilateral conjugate gaze palsy and nystagmus (medial longitudinal fasciculus); ipsilateral impaired eye abduction (abducens nucleus); ipsilateral hemiataxia (middle cerebellar peduncle); and contralateral hemisensory loss (lateral spinothalamic tract and medial lemniscus) (Saposnik, G. 2008; Elyas, AE. 2011).

Multiple pontine infarcts primarily effecting the perforating arteries' territories result in pseudobulbar palsy due to the involvement of the corticobulbar fibers. A pseudobulbar palsy presentation includes severe dysphagia and dysarthria. Some individuals may present with pathological crying or laughing (Saposnik, G. 2008; Elyas, AE. 2011).

Bilateral pontine infarcts happen secondary to impeded blood flow in the larger basilar artery. Being bilateral, both left and right, upper, and lower limbs can be involved (tetraplegia) and also impair the consciousness. Large infarcts affecting the corticospinal, corticobulbar, and corticopontine tracts result in the locked-in syndrome. Locked-in syndrome presents with tetraplegia, bilateral facial paralysis, pharyngeal, and horizontal gaze palsy with retained consciousness and cognitive abilities. Recurrent stereotypical episodes of dysarthria, ophthalmoplegia, motor, or sensory disturbances are more recently referred to as the "pontine warning syndrome." It is hypothesized that this syndrome can progress into a bilateral pontine infarct (Saposnik, G. 2008; Elyas, AE. 2011).

The pons is part of the brainstem that relays neural signals from the cerebrum and cerebellum to organs all over the body. Further, cranial nerves V-VIII originate from the pons which control involuntary vital centers, breathing (intensity and frequency), and the sleepwake cycle. Thus, massive pathology at the pons may indicate a poor prognosis due to these important functions. Primary pontine hemorrhage (PPH) accounts for approximately $5 \%-$ $10 \%$ of intracranial hemorrhages, and the overall mortality rates in recent studies were $40 \%$ 
$50 \%$. In general, patients with $\mathrm{PPH}$ are treated conservatively. Many reports of massive pontine stroke, either by hemorrhage or infarction, showed high morbidity and mortality rates. Many factors affect the prognosis of PPH, especially the level of consciousness and size of the hematoma. This report shows a good clinical outcome following adequate treatment after the onset of an acute massive pontine hemorrhage (Sripontan, S. 2019; Jang, JH. 2010; Behrouz, R. 2018).

Initial assessment of an individual presenting with stroke symptoms should include blood pressure and heart rate measurement along with respiratory function assessment (respiratory rate and pulse oximetry). As pons houses the respiratory centers, large pontine infarctions can compromise respiratory function and may warrant intubation and mechanical ventilation. If hemodynamically stable, a complete neurological examination in addition to routine history taking and physical examination is conducted. While the neurological exam assists in localizing the anatomical region affected and the type of stroke (ischemic vs. hemorrhagic), relevant history and vitals can point to the predisposing conditions (e.g., hypertension). After proper stabilization, a complete neurological examination including an assessment of consciousness, cognition, gait, coordination, reflexes, cranial nerve examination, and sensory and the motor system should be performed. Followed by physical examination, neuroimaging is urgently indicated in all suspected stroke cases, and the timing of the imaging determines the treatment modality and its success. Computed tomography (CT) scan due to its availability and ability to detect hemorrhages is used to triage a stroke patient. However, magnetic resonance imaging (MRI) remains the mainstay of diagnosis for ischemic strokes due to its ability to detect acute ischemia within minutes of onset (diffusion-weighted MRI). Neurovascular imaging (CT or MR angiography) is performed in those with large artery obstruction (e.g., basilar artery) as they might have to undergo mechanical thrombectomy. In cases where an embolus is suspected, imaging studies like carotid doppler or transthoracic echocardiography may be required to identify the degree of stenosis or the source of embolus. Basic investigations like complete blood count, serum glucose, serum electrolytes, renal and liver function tests, lipid profile, and a 12-lead electrocardiogram are routinely ordered. Various other biomarkers (e.g., c-Fn, MMP-9) are being investigated as possible predictors of stroke outcomes and can very well be recommended as routine tests for all stroke patients in the future (Chernov, D. 2019; Kamo, H. 2019; Zhang, H-T. 2018; Yilmaz, TF. 2017).

Based on the available evidence, the overall all-cause mortality rate for $\mathrm{PH}$ is roughly $50 \%$. Level of consciousness on admission and haemorrhage size are the strongest and most consistent predictors of mortality in patients with PH. Small PH with dorsal and lateral localisations may have better survival rates, compared to other localisations; certainly, more favourable prognosis than massive PH. Age, IVE and hydrocephalus were not consistently found to be strong prognostic features. No study to date has investigated the effect of haemorrhage growth specifically in PH (Zhang, M. 2018; Xia, N-G. 2018).

Over the four decades in which these studies were conducted, the survival of $\mathrm{PH}$ appears to have improved. This may be partly due to advancement is the fields of neurology, neurosurgery and intensive care medicine. Improved public perception of ICH and stroke in general may have contributed to this progress. However, it is not clear if functional outcomes have improved in parallel to survival (Karadan, U. 2018; Hao, G. 2019).

The diagnosis and management of brainstem stroke bring a considerable burden to the healthcare system, the patient, the family members, and the society at large. The slow increase in the global burden of stroke has been steadily increasing (Chang, MC. 2018). 


\section{Conclusion}

The enhancement must start with proper patient education about the risk factors and how they can be modified. A simple community educational approach about smoking cessation, a healthy diet, an active lifestyle, regular health screening for diabetes mellitus and hypertension, drug addiction cessation, and rehabilitation can be undertaken. A decentralized model where a community-level assessment of primary and secondary prevention of noncommunicable disease can result in a reduction in the incidence of stroke (Ling, L. 2009; Burson K. 2020).

\section{References}

Behrouz R. Prognostic factors in pontine haemorrhage: A systematic review. Eur stroke J. 2018/01/08. 2018 Jun;3(2):101-9.

Burson K, Mastenbrook J, Van Dommelen K, Shah M, Bauler LD. A Focal Pontine Infarct Presenting as Unilateral Facial Nerve Paralysis. Cureus. 2020 Sep 25;12(9):e10646e10646.

Chang MC, Kwak SG, Chun MH. Dysphagia in patients with isolated pontine infarction. Neural Regen Res. 2018 Dec;13(12):2156-9.

Chernov D, Karavassilis ME, Hassan F, Bhandari M. Bilateral paramedian pontine infarcts: a rare cause of bilateral horizontal gaze palsy. BMJ Case Rep. 2019 Oct;12(10).

Ekerikevwe, K.I. (2020). Survival Analysis of the Clinical Episodes of Stroke Using Data from

Western Delta Region of Nigeria. Britain International of Exact Sciences (BIoEx) Journal Vol. 2 (1):1-10.

Elyas AE, Bulters DO, Sparrow OC. Pathological laughter and crying in patients with pontine lesions. J Neurosurg Pediatr. 2011 Dec;8(6):544-7.

Hao G, Xu Z, Zhu J. Surgical treatment of spontaneous brainstem hemorrhage: A case report. Medicine (Baltimore). 2019 Dec;98(51):e18430.

Jang JH, Song YG, Kim YZ. Predictors of 30-day mortality and 90-day functional recovery after primary pontine hemorrhage. J Korean Med Sci. 2010/12/22. 2011 Jan;26(1):100-7.

Jillella. GMD V. Pontine Infarction - StatPearls - NCBI Bookshelf. 2021. p. Treasure Island (FL): StatPearls Publishing; 2021.

Jesus. SNGO De. Brainstem Infarction - StatPearls - NCBI Bookshelf. 2021. p. Treasure Island (FL): StatPearls Publishing; 2021.

Kamo H, Ueno Y, Sugiyama M, Miyamoto N, Yamashiro K, Tanaka R, et al. Pontine hemorrhage accompanied by neuromyelitis optica spectrum disorder. J Neuroimmunol. 2019 May;330:19-22.

Karadan U, Supreeth RN, Manappallil RG, Jayakrishnan C. Twenty-Four Syndrome: An Untold Presentation of Pontine Hemorrhage. J stroke Cerebrovasc Dis Off J Natl Stroke Assoc. 2018 May;27(5):e73-4.

Ling L, Zhu L, Zeng J, Liao S, Zhang S, Yu J, et al. Pontine infarction with pure motor hemiparesis or hemiplegia: a prospective study. BMC Neurol. 2009 Jun 15;9:25.

Naqvi. OKDLMIA. Foville Syndrome - Stat Pearls - NCBI Bookshelf. 2020. p. Treasure Island (FL): StatPearls Publishing; 2021.

Saposnik G, Noel de Tilly L, Caplan LR. Pontine warning syndrome. Arch Neurol. 2008 Oct; 65(10):1375-7.

Sari, A.P., Lubis, R., and Zulfendri. (2020). Determinant of Hypertension, Obesity and Smoking on Stroke Events in Hospital Patients at Regional General Hospitalof Rokan Hulu Riau District, 2018 (Epidemiology and Public Health). Britain International of 
Exact Sciences (BIoEx) Journal Vol. 2 (1):384-389.

Sripontan S. Good Outcome in a Patient with Massive Pontine Hemorrhage. Asian J Neurosurg. 2019;14(3):992-5.

Wei Y, Wu L, Wang Y, Liu J, Miao P, Wang K, et al. Disrupted Regional Cerebral Blood Flow and Functional Connectivity in Pontine Infarction: A Longitudinal MRI Study. Front Aging Neurosci. 2020 Nov 19;12:577899.

Xia N-G, Chen Y-Y, Li J, Chen X, Ye Z-S, Chen S-Y, et al. Eight-and-a-half syndrome caused by a pontine haemorrhage: a case report and review of the literature. Int J Neurosci. 2018 Aug;128(8):746-50.

Yllmaz TF, Aralasmak A, Toprak H, Kocaman G, Alkan A. Pontine bleeding following drainage of subdural hematoma in craniospinal hypotension. Radiol case reports. 2017 Oct 31;13(1):228-32.

Zhang H-T, Chen L-H, Bai M-C, Xu R-X. Anterior subtemporal approach for severe upper pontine hematomas: A report of 28 surgically treated cases. J Clin Neurosci Off J Neurosurg Soc Australas. 2018 Aug;54:20-4.

Zhang M, Liu R, Xing H, Luo H, Cui L, Sun Z. A Simple and Rapid Puncture Method for Draining Hematoma in Pontine Hemorrhage. Front Neurol. 2018 Nov 19;9:794. 